Pacific Journal of Mathematics

A CHARACTERIZATION OF THE FINITE MOUFANG 


\section{A CHARACTERIZATION \\ OF THE FINITE MOUFANG HEXAGONS BY GENERALIZED HOMOLOGIES}

\section{H. VAN MALDEghem}

A generalized homology of a generalized hexagon $\mathscr{S}$ is an automorphism of $\mathscr{S}$ fixing all points on two mutually opposite lines or fixing all lines through two mutually opposite points. We show that if $\mathscr{S}$ is finite and if it admits "many" generalized homologies, then $\mathscr{S}$ is Moufang and hence classical.

1. Introduction and notation. A (finite thick) generalized hexagon of order $(s, t)$ is a point-line incidence geometry $\mathscr{S}=(P, B, I)$ satisfying ( $\mathrm{GH} 1)$ up to $(\mathrm{GH} 4)$.

(GH 1) There are $s+1$ points incident with each line, $s>1$.

(GH 2) There are $t+1$ lines incident with each point, $t>1$.

(GH 3) Every two varieties (a variety is a point or a line) lie in a common circuit consisting of six points and six lines.

(GH 4) For every circuit consisting of $k$ points and $k$ lines it must be that $k \geq 6$.

At present there are, up to duality, only two classes known of finite generalized hexagons and they are related to the Chevalley groups $G_{2}(q)$ and ${ }^{3} D_{4}(q)$. We denote them respectively by $G_{2}(q)$ and ${ }^{3} D_{4}(q)$ (see e.g. [4]). Of course, there are two mutually dual choices for these generalized hexagons, but we fix one by saying that ${ }^{3} D_{4}(q)$ has order $\left(q, q^{3}\right)$ and $G_{2}(q)$ is a subgeometry of ${ }^{3} D_{4}(q)$. We will define these hexagons below using Kantor's description (see [4]).

We now introduce some further notation. Let $\mathscr{S}=(P, B, I)$ be a finite generalized hexagon. We will always assume that $\mathscr{S}$ is thick. A circuit consisting of six points and six lines (as in (GH 3)) is called an apartment. Let $A$ be an apartment and $x$ a variety of $\mathscr{S}$. We denote the set of all varieties incident with $x$ but distinct from the 12 varieties of $A$ by $A^{*}(x)$. A chain of seven distinct consecutively incident varieties is called a root. If the middle element of a root is a point, then we call the root short, if the middle element is a line, then we call it a long root. Let $\mathfrak{R}=\left(x_{0} I x_{1} I \cdots I x_{6}\right)$ be a root. If $\alpha$ is an automorphism of $\mathscr{S}$ fixing all varieties incident with $x_{1}, x_{2}, x_{3}$, 
$x_{4}$ or $x_{5}$, then we call $\alpha$ an $\mathfrak{R}$-elation or, in general, a root-elation. If the group of $\mathfrak{R}$-elations acts transitively on the set of apartments containing $\mathfrak{R}$ (for fixed $\mathfrak{R}$ ), then we call $\mathfrak{R}$ a transitive root. In that case the action just mentioned is regular. If every root of $\mathscr{S}$ is transitive, then $\mathscr{S}$ is called Moufang and it was observed by Tits (see [9]) that a theorem of Fong and Seitz [3] implies that, amongst other things, all finite Moufang generalized hexagons arise from the Chevalley groups mentioned above.

Two varieties $x$ and $y$ of $\mathscr{S}$ are called opposite if they lie at distance 6 from each other in the incidence graph, i.e. if they are opposite vertices or opposite sides in every apartment containing them. Let $x$ and $y$ be two opposite varieties and let $\alpha$ be an automorphism of $\mathscr{S}$ fixing every variety incident with $x$ or $y$. Then we call $\alpha$ a generalized homology or an $(x, y)$-homology. Consider the group $\mathscr{H}(x, y)$ of all $(x, y)$-homologies. The number of orbits of $\mathscr{H}(x, y)$ on the set of all varieties incident with a given variety $z$ which is in turn incident with $x$ or $y$ is independent of the choice of $z$ and it is at least 3 (since $\{x\}$ or $\{y\}$ is an orbit, as is the unique variety incident with $z$ and nearest to $x$ or $y$ ). If that number is exactly 3 for some (and hence for all) such $z$, then we say that $\mathscr{S}$ is $(x, y)$-transitive and that $(x, y)$ is a transitive pair. If every pair of opposite varieties of a given apartment $A$ is transitive, then we call $A$ itself transitive. If every apartment of $\mathscr{S}$ is transitive, then we say that $\mathscr{S}$ has transitive apartments. The aim of the present paper is to show that the latter is equivalent to $\mathscr{S}$ being Moufang. Hence our main result:

Main Result. A finite thick generalized hexagon $\mathscr{S}$ is Moufang if and only if it has transitive apartments. If $\mathscr{S}$ has order $(s, t)$ with $s>$ 2 and $t>2$ and $\mathscr{S}$ is Moufang, then all root-elations are generated by generalized homologies.

There is an immediate corollary.

COROLlARY 1. A finite thick generalized hexagon $\mathscr{S}$ has transitive apartments if and only if $\mathscr{S}$ or its dual is isomorphic to $G_{2}(q)$ or to ${ }^{3} D_{4}(q)$ for some prime power $q$.

In $\S 2$ we will show that both $G_{2}(q)$ and ${ }^{3} D_{4}(q)$ have transitive apartments (and hence also their duals). In $\S 3$ we prove the converse.

2. The classical generalized hexagons $G_{2}(q)$ and ${ }^{3} D_{4}(q)$. We start with Kantor's description of ${ }^{3} D_{4}(q)$ (see [4]). 
Let

$$
\begin{aligned}
& Q=\left\{(a, \beta, c, \delta, e) \mid a, c, e \in G F(q) ; \beta, \delta \in G F\left(q^{3}\right)\right\}, \quad \text { and } \\
& (a, \beta, c, \delta, e)\left(a^{\prime}, \beta^{\prime}, c^{\prime}, \delta^{\prime}, e^{\prime}\right) \\
& \quad=\left(a+a^{\prime}, \beta+\beta^{\prime}, c+c^{\prime}+a^{\prime} e-\operatorname{tr}\left(\beta^{\prime} \delta\right), \delta+\delta^{\prime}, e+e^{\prime}\right)
\end{aligned}
$$

where $\operatorname{tr}(\gamma)=\gamma+\gamma^{q}+\gamma^{q^{2}}$. So $Q$ is a group of order $q^{9}$. If $1 \leq i \leq 5$, let $x_{i}$ be the element whose $i$ th coordinate is $x$ and all others 0 , and let $X_{i}$ be the set of all such $x_{i}$. Define for all $x \in G F\left(q^{3}\right)$ an automorphism $x_{6}$ of $Q$ by

$$
\begin{aligned}
& (a, \beta, c, \delta, e)^{x_{6}} \\
& =\left(a, \beta+a x, c-a^{2} x^{1+q+q^{2}}-\operatorname{tr}\left(\beta^{q+q^{2}} x\right)-\operatorname{tr}\left(a \beta x^{q+q^{2}}\right),\right. \\
& \quad \delta+a x^{q+q^{2}}+\beta^{q} x^{q^{2}}+\beta^{q^{2}} x^{q}, \\
& \left.e+a x^{1+q+q^{2}}+\operatorname{tr}\left(\beta x^{q+q^{2}}\right)+\operatorname{tr}(\delta x)\right) .
\end{aligned}
$$

Now identify $t \in G F(q)$ with $t_{6}$ and define

$$
\begin{array}{ll}
A_{1}(\infty)=X_{5}, & A_{1}(t)=X_{1}^{t}, \\
A_{2}(\infty)=X_{4} X_{5}, & A_{2}(t)=\left(X_{1} X_{2}\right)^{t}, \\
A_{3}(\infty)=X_{3} X_{4} X_{5}, & A_{3}(t)=\left(X_{1} X_{2} X_{3}\right)^{t}, \\
A_{4}(\infty)=X_{2} X_{3} X_{4} X_{5}, & A_{4}(t)=\left(X_{1} X_{2} X_{3} X_{4}\right)^{t} .
\end{array}
$$

Now let $t$ run over $G F\left(q^{3}\right) \cup\{\infty\}$ and $g$ over $Q$. Then the points of ${ }^{3} D_{4}(q)$ are a symbol $(\infty)$, all possible cosets $A_{4}(t) g$ and $A_{2}(t) g$, and all elements $g$. The lines of ${ }^{3} D_{4}(q)$ are the elements $t$ and the cosets $A_{3}(t) g$ and $A_{1}(t) g$. Incidence is obtained via (suitable) inclusion and moreover $t$ is incident with $A_{4}(t) g$ and also with $(\infty)$.

Restricting $\beta, \delta$ and $t$ above to $G F(q)$ produces $G_{2}(q)$.

Now we fix the following apartment $A$ in ${ }^{3} D_{4}(q)$.

$$
\begin{array}{r}
A=\left(A_{4}(\infty) I A_{3}(\infty) I A_{2}(\infty) I A_{1}(\infty) I(0,0,0,0,0) I A_{1}(0)\right. \\
\left.I A_{2}(0) I A_{3}(0) I A_{4}(0) I 0 I(\infty) I \infty I A_{4}(\infty)\right) .
\end{array}
$$

Let $T \in G F\left(q_{3}\right)$ and define the following automorphism $\theta_{T}$ of $Q$.

$$
\theta_{T}:(a, \beta, c, \delta, e) \rightarrow\left(a, T \beta, T^{1+q+q^{2}} c, T^{q+q^{2}} \delta, T^{1+q+q^{2}} e\right) .
$$

Define $\theta_{T}$ also on the group of automorphsims $t_{6}$ by mapping $t_{6} \rightarrow$ $(T t)_{6}$. Then it is an exercise to show that the mapping $\theta_{T}$ produces an automorphism of ${ }^{3} D_{4}(q)$ leaving all elements of $A$ and all points incident with the line $\infty$ invariant and mapping the line $t$ into the line $T t$. So we obtain a group of order $q^{3}-1$ acting transitively 
on $A^{*}((\infty))$. This group is isomorphic to the multiplicative group of $G F\left(q^{3}\right)$. Hence ${ }^{3} D_{4}(q)$ is $(x, y)$-transitive for all pairs $(x, y)$ of opposite points (since the full automorphism group of ${ }^{3} D_{4}(q)$ acts transitively on such pairs).

Now let $U \in G F(q)$ and define the following automorphism $\eta_{U}$ of $Q$.

$$
\eta_{U}:(a, \beta, c, \delta, e) \rightarrow\left(U a, U \beta, U^{2} c, U \delta, U e\right) .
$$

Then this induces an automorphism of ${ }^{3} D_{4}(q)$ leaving all elements of $A$ and all lines incident with the point $(\infty)$ invariant and mapping the point $(a, 0,0,0,0)$ into the point $(U a, 0,0,0,0)$. As above, we conclude that ${ }^{3} D_{4}(q)$ is $(x, y)$-transitive for all pairs $(x, y)$ of opposite lines. Hence ${ }^{3} D_{4}(q)$ has transitive apartments.

Restricting $\beta, \delta, t$ and $T$ to $G F(q)$, we see that also $G_{2}(q)$ has transitive apartments. This shows one way of our main result.

\section{Finite generalized hexagons with transitive apartments.}

3.1. Generalities. From now on we fix a given finite thick generalized hexagon $\mathscr{S}$ of order $(s, t)$ and a certain apartment $A$ in $\mathscr{S}$. We suppose that $\mathscr{S}$ has transitive apartments. By duality, we can assume that $s \geq t$. We denote the elements of $A$ by

$$
L_{1} I p_{1} I L_{2} I p_{3} I L_{4} I p_{5} I L_{6} I p_{6} I L_{5} I p_{4} I L_{3} I p_{2} I L_{1} \text {. }
$$

If $x_{1}, x_{2}, \ldots, x_{i}, i$ a positive integer, are varieties of $\mathscr{S}$ then we denote by $\mathscr{H}\left(x_{1}, x_{2}, \ldots, x_{i}\right)$ the group of automorphisms of $\mathscr{S}$ fixing all varieties incident with at least one of $x_{1}, \ldots, x_{i}$. If we want the group fixing moreover varieties $y_{1}, y_{2}, \ldots, y_{j}$ for some positive integer $j$, then we write $\mathscr{H}_{\left(y_{1}, y_{2}, \ldots, y_{j}\right)}\left(x_{1}, \ldots, x_{i}\right)$. We denote the identity of the automorphism group of $\mathscr{S}$ by the usual 1 . Here are some useful lemmas.

Lemma 1. Let $p \in\left\{p_{1}, p_{3}\right\}, L \in\left\{L_{1}, L_{2}, L_{4}\right\}$ and $p I L$. If $\mathscr{H}_{\left(p_{2}, p_{s}\right)}(p, L) \neq 1$, then $\left|\mathscr{H}_{\left(p_{2}, p_{5}\right)}(p, L)\right|=t$. Also the dual holds.

Proof. This is obvious if $t=2$, so suppose $t \neq 2$. Let $1 \neq \sigma \in$ $\mathscr{H}_{\left(p_{2}, p_{5}\right)}(p, L)$ and $\alpha \in \mathscr{H}\left(L_{1}, L_{6}\right)$. Consider $\alpha^{-1} \sigma \alpha$. Then $L_{3}^{\alpha^{-1} \sigma \alpha}=$ $\left(L_{3}^{\sigma}\right)^{\alpha}$ runs over all elements of $A^{*}\left(p_{2}\right)$ as $\alpha$ varies over $\mathscr{H}\left(L_{1}, L_{6}\right)$. But clearly $\alpha^{-1} \sigma \alpha \in \mathscr{H}_{\left(p_{2}, p_{5}\right)}(p, L)$.

LEMMA 2. If $s>2$ and $t>2$, then the group of automorphisms of $\mathscr{S}$ generated by all generalized homologies of $\mathscr{S}$ acts transitively on 
the triplets $(p, L, B)$ where $p$ is a point incident with the line $L$ and both lying in the apartment $B$.

Proof. It is easy to see that the group in question acts transitively on the set of points opposite to a fixed point, hence it acts transitively on the set of points. Dually, it acts transitively on the set of lines and even on the set of pairs $(p, L)$ of points $p$ incident with a line $L$. If $B$ and $B^{\prime}$ are two apartments containing such a pair $(p, L)$, then it is again elementary to see that we can map $B^{\prime}$ to $B$ fixing $(p, L)$.

Lemma 3. Suppose $s>2$. Then we have:

(a) Suppose $\mathscr{S}$ does not contain a proper thick subhexagon with $s+1$ points on a line. Then $\mathscr{H}_{p_{5}}\left(p_{1}, L_{1}\right) \neq 1$. If moreover $\mathscr{H}\left(L_{1}, L_{6}\right)=$ $\mathscr{H}\left(L_{2}, L_{5}\right)$, then $\mathscr{H}\left(L_{1}, L_{6}\right)=\mathscr{H}\left(L_{3}, L_{4}\right)$ and every long root is transitive. On the other hand, if moreover $\mathscr{H}\left(L_{1}, L_{6}\right)$ acts regularly on $A^{*}\left(L_{2}\right)$, then $\mathscr{H}_{p_{5}}\left(L_{1}, p_{1}, L_{2}\right) \neq 1$. Also the dual holds.

(b) Suppose $\mathscr{H}\left(L_{2}, L_{5}\right)$ acts regularly on $A^{*}\left(p_{2}\right)$.

Then $\mathscr{L}_{\left(p_{2}, p_{5}\right)}\left(p_{1}, L_{2}\right) \neq 1$. Also the dual holds.

Proof. (a) Let $\left(p_{2} I M_{3} I q_{4} I M_{5} I q_{6} I M_{6} I p_{5}\right)$ be a (long) root not lying in $A$. Let $1 \neq \alpha \in \mathscr{H}\left(L_{1}, L_{6}\right)$ and choose $\beta \in \mathscr{H}\left(L_{1}, M_{6}\right)$ such that $\alpha \beta$ fixes at least one element of $A^{*}\left(p_{1}\right)$. Clearly $\alpha \beta \neq$ 1. Suppose $\alpha \beta$ fixes a line $M$ incident with $p_{2}$ and distinct from $L_{1}$. Then it fixes a whole apartment, all points incident with $L_{1}$ and at least three lines through $p_{1}$; hence it fixes a thick subhexagon with $s+1$ points on a line. By assumption this implies $\alpha \beta=1$, a contradiction. Suppose now $\alpha \beta$ does not fix a line $M$ incident with $p_{1}$. Let $L$ be any element of $A^{*}\left(p_{5}\right)$ and consider the $\left(L_{1}, L\right)$ homology $\alpha_{L}$ mapping $L_{6}$ into $L_{6}^{\alpha \beta}$. If also $L^{\prime} \in A^{*}\left(p_{5}\right)$, then $\alpha_{L} \alpha_{L^{\prime}}^{-1}$ is an $\left(L_{1}, L_{6}\right)$-homology and hence, if $L \neq L^{\prime}$, then $\alpha_{L} \alpha_{L^{\prime}}^{-1}$ does not fix $M$, so $M^{\alpha_{L}} \neq M^{\alpha_{L^{\prime}}}$. But there are $t-2$ valid choices for $L$, so $\left\{M^{\alpha_{L}}\right\}$ contains the $t-2$ lines incident with $p_{1}$ and distinct from $M, L_{1}$ and $L_{2}$. Hence we can choose $L$ such that $\alpha_{L}$ maps $M$ into $M^{\alpha \beta}$. But similarly as before, $\alpha \beta \alpha_{L}^{-1}$ fixes $A$, it fixes all points incident with $L_{1}$ and it fixes $M$; hence it is the identity, contradicting the fact that $\alpha \beta$ does not fix $L$. Hence $\alpha \beta$ must fix all lines incident with $p_{1}$. But $\alpha \beta$ fixes $p_{5}$, and hence the first assertion follows.

Suppose now $\mathscr{H}\left(L_{1}, L_{6}\right)=\mathscr{H}\left(L_{2}, L_{5}\right)$. Then by Lemma 1, $\mathscr{H}\left(L_{1}, L_{6}\right)=\mathscr{H}\left(L_{3}, L_{4}\right)$. So $\alpha \beta$ above fixes also all points incident with $L_{2}$ or $L_{4}$. But since $\alpha \beta$ acts semi-regularly on the lines incident with $p_{2}$ (distinct from $L_{1}$ ), it has to fix at least one line 
$M^{\prime} \in A^{*}\left(p_{3}\right)$. By symmetry and by Lemma 1 , there exists an automorphism $\sigma \in \mathscr{H}\left(L_{1}, L_{2}, p_{3}, L_{4}\right)$ mapping $L_{3}$ into $L_{3}^{\alpha \beta}$. So $\alpha \beta \sigma^{-1}$ fixes $A, M^{\prime}$ and all points of $L_{1}$; hence it fixes $\mathscr{S}$. So $\alpha \beta=\sigma$, and hence $\alpha \beta \in \mathscr{H}\left(L_{1}, p_{1}, L_{2}, p_{3}, L_{4}\right)$. The second assertion now follows from Lemma 1.

Similar to the first assertion, one shows that every element of $\mathscr{Z}_{p_{5}}\left(p_{1}, L_{1}\right)$ must also fix all points of $L_{2}$.

(b) This is similar to the first part of (a).

Lemma 4. Suppose $p \in\left\{p_{1}, p_{2}, p_{4}\right\}$ and $L \in\left\{L_{1}, L_{3}\right\}$. Suppose both groups $\mathscr{H}\left(p, p^{\prime}\right)$ and $\mathscr{H}\left(L, L^{\prime}\right)$, where $p^{\prime}$ is opposite to $p$ in $A$ and $L^{\prime}$ is opposite to $L$ in $A$, act non-trivially on $A^{*}\left(L_{2}\right)$ and at least one of them acts (modulo the kernel of the action) semi-regularly on $A^{*}\left(L_{2}\right)$. Then $\mathscr{H}_{\left(L_{2}, L_{5}\right)}(p, L)$ contains non-trivial elements. If moreover $\mathscr{H}\left(p, p^{\prime}\right)$ or $\mathscr{H}\left(L, L^{\prime}\right)$ contains non-trivial automorphisms fixing $A^{*}(x)$ elementwise, for some variety $x \in\left\{p_{1}, L_{1}, p_{2}, L_{3}, p_{4}\right\}$, then $\mathscr{H}_{\left(L_{2}, L_{5}\right)}(p, L, x)=\mathscr{H}_{\left(L_{2}, L_{5}\right)}(p, L)$. Also the dual holds.

Proof. Let $q_{3} \in A^{*}\left(L_{2}\right)$ and suppose $q_{3} I M_{4} I q_{5} I M_{6} I q_{6} I L_{5}$. Choose $\alpha \in \mathscr{H}\left(p, p^{\prime}\right)$ not fixing $q_{3}$ and $\beta \in \mathscr{H}\left(L, L^{\prime \prime}\right)$ not fixing $p_{3}$, where $L^{\prime \prime} \in\left\{M_{4}, M_{6}\right\}$ is opposite to $L$ (this can be done by assumption (use also Lemma 2) possibly by taking a "new" point $q_{3}$ ). Put $\sigma=\alpha^{-1} \beta \alpha \beta^{-1}$. Clearly $\sigma \in \mathscr{H}_{\left(L_{2}, L_{5}\right)}(p, L)$. Suppose $\sigma$ is the identity. Then $p_{3}^{\sigma}=p_{3}$; hence $\left(p_{3}^{\beta}\right)^{\alpha}=p_{3}^{\beta}$ and similarly $\left(q_{3}^{\alpha}\right)^{\beta}=q_{3}^{\alpha}$. But this contradicts the semi-regularity of $\mathscr{H}\left(p, p^{\prime}\right)$ or $\mathscr{H}\left(L, L^{\prime \prime}\right)$ on the appropriate set of points. Hence the first assertion. The second assertion now follows easily by the construction of $\sigma$ above and by Lemma 1.

LEMMA 5. Let $\sigma$ be an automorphism of $\mathscr{S}$ fixing $L_{2}, p_{1}, L_{1}, p_{2}$, $L_{3}, p_{4}, L_{5}$ and acting semi-regularly on the set of points distinct from $p_{1}$ incident with $L_{2}$. Let $x \in\left\{p_{1}, L_{1}, p_{2}, L_{3}, p_{4}\right\}$ and suppose $\mathscr{H}(x, y)$, where $y$ is opposite to $x$ in $A$, acts non-trivially on $A^{*}\left(L_{2}\right)$. Then there exists a non-trivial automorphism $\tau$ of $\mathscr{S}$ fuxing all varieties incident with $x$, fixing $L_{2}, p_{1}, \ldots, L_{5}$ and fixing $L_{1}$ or $L_{3}$ pointwise and $p_{1}, p_{2}$ or $p_{4}$ linewise whenever $\sigma$ does. Also the duat holds.

Proof. Let $\alpha$ be an $(x, y)$-homology acting non-trivially on $A^{*}\left(L_{2}\right)$. Possibly by replacing $\sigma$ by $\beta^{-1} \sigma \beta$ for some $\beta \in \mathscr{H}\left(p_{1}, p_{6}\right)$, we see 
that $\alpha$ does not fix $p_{3}^{\sigma}$. Defining $\tau=\alpha^{-1} \sigma \alpha \sigma^{-1}$, the assertion follows similarly as in the proof of the previous lemma.

From here on, we have to distinguish between three different cases: $s=t ; s>t$ and $\mathscr{S}$ contains a subhexagon of order $\left(s^{\prime}, t\right), 1<s^{\prime}<$ $s ; s>t$ and $\mathscr{S}$ does not contain any subhexagon of order $\left(s^{\prime}, t\right)$ for any $1<s^{\prime}<s$.

3.2. The case $s=t$. Suppose $s=t$. By Cohen-Tits [1] (see also Tits [8]), we may assume $s>2$; otherwise $\mathscr{S}$ is Moufang. If some non-trivial $\alpha \in \mathscr{H}\left(p_{1}, p_{6}\right)$ fixes some point $p \in A^{*}\left(L_{1}\right)$, then it fixes a subhexagon of order $\left(s^{\prime}, s\right), 1<s^{\prime}$ and hence by a theorem of Thas [6], $s^{2} \geq s^{\prime 2} s^{2}$, contradicting $s^{\prime}>1$. Hence $\mathscr{H}\left(p_{1}, p_{6}\right)$ acts regularly on $A^{*}\left(L_{1}\right)$. Dually, $\mathscr{H}\left(L_{1}, L_{6}\right)$ acts regularly on $A^{*}\left(p_{1}\right)$. By Lemma 3, the groups $\mathscr{H}_{L_{5}}\left(p_{1}, L_{1}\right)$ and $\mathscr{H}_{p_{5}}\left(p_{1}, L_{1}\right)$ are non-trivial.

Suppose first that $\mathscr{H}\left(L_{1}, L_{6}\right)=\mathscr{H}\left(p_{3}, p_{4}\right)$. Then, by the above argument, $\mathscr{H}\left(L_{1}, L_{6}\right)$ acts regularly on $A^{*}\left(L_{2}\right)$ and hence by Lemma 3, $\mathscr{H}_{p_{5}}\left(L_{1}, p_{1}, L_{2}\right)$ is non-trivial. Dually, one shows that also $\mathscr{H}_{L_{5}}\left(p_{1}, L_{1}, p_{2}\right)$ is non-trivial. By [10], $\mathscr{S}$ is Moufang.

Hence we may assume that $\mathscr{H}\left(L_{1}, L_{6}\right) \neq \mathscr{H}\left(p_{3}, p_{4}\right)$. Suppose $\mathscr{H}\left(p_{1}, p_{6}\right)=\mathscr{H}\left(p_{2}, p_{5}\right)$. By Lemma 3 , all short roots are transitive. Suppose $\alpha \in \mathscr{H}\left(L_{2}, L_{5}\right)$ fixes some $M \in A^{*}\left(p_{2}\right)$. Let $p$ be any element of $A^{*}\left(L_{1}\right)$ and let $\beta \in \mathscr{H}\left(p_{1}, p_{6}\right)$ be such that $p^{\alpha}=p^{\beta}$. Then $\alpha \beta^{-1}$ fixes $A, M$ and $p$; hence it fixes a thick subhexagon of $\mathscr{S}$. So there exsits a point $p^{\prime} \in A^{*}\left(L_{1}\right)$ fixed by $\alpha \beta^{-1}$ (since $L_{2}$ belongs to that subhexagon). So clearly $\beta$ must fix $p$, a contradiction. Hence $\mathscr{H}\left(L_{2}, L_{5}\right)$ acts regularly on $A^{*}\left(p_{2}\right)$. By Lemma $3, \mathscr{H}_{\left(p_{2}, p_{5}\right)}\left(p_{1}, L_{2}\right)$ is not trivial. By Lemma 1 and Van Maldeghem-Weiss [10], $\mathscr{S}$ is Moufang. Similarly $\mathscr{S}$ is Moufang if $\mathscr{H}\left(L_{1}, L_{6}\right)=\mathscr{H}\left(L_{2}, L_{5}\right)$.

By the preceding paragraph, we may assume $\mathscr{H}\left(L_{1}, L_{6}\right) \neq$ $\mathscr{H}\left(L_{2}, L_{5}\right), \mathscr{H}\left(p_{1}, p_{6}\right) \neq \mathscr{H}\left(p_{2}, p_{5}\right)$ and $\mathscr{H}\left(L_{1}, L_{6}\right) \neq \mathscr{H}\left(p_{3}, p_{4}\right)$. So the group $\mathscr{H}\left(p_{1}, p_{6}\right)$ acts non-trivially on $A^{*}\left(p_{2}\right)$. But if a nonidentity element $\alpha \in \mathscr{H}\left(p_{1}, p_{6}\right)$ fixes at least one element of $A^{*}\left(p_{2}\right)$, then it must fix a subhexagon of order $(1, s)$ and hence $\alpha$ fixes all elements of $A^{*}\left(p_{2}\right)$. So up to its kernel, the action of $\mathscr{H}\left(p_{1}, p_{6}\right)$ on $A^{*}\left(p_{2}\right)$ is non-trivial and semi-regular. By Lemma 4, the group $\mathscr{H}_{\left(p_{2}, p_{5}\right)}\left(p_{1}, L_{2}\right)$ is non-trivial. Dually, the group $\mathscr{H}_{\left(L_{2}, L_{5}\right)}\left(p_{2}, L_{1}\right)$ is non-trivial. By Lemma 1 and Van Maldeghem-Weiss [10], $\mathscr{S}$ is Moufang.

Clearly, there follows from our proof that, if $s>2$ and $t>2$, the set of generalized homologies generate all root-elations. This completes the case $s=t$. 
3.3. Case $s>t$ and $\mathscr{S}$ contains a subhexagon of order $\left(s^{\prime}, t\right)$, $1<s^{\prime}<s$. In this case, we can assume by Lemma 2 that there is a subhexagon $\mathscr{S}^{\prime}$ of order $\left(s^{\prime}, t\right)$ containing $A$. Let $p$ be a point of $\mathscr{S}^{\prime}$ incident with $L_{1}$. Consider $\alpha \in \mathscr{H}\left(p_{1}, p_{6}\right)$ mapping $p$ into a point $p^{\prime}$ not lying in $\mathscr{S}^{\prime}$. Then $S^{\prime \alpha} \cap \mathscr{S}^{\prime}$ is a proper subhexagon of $\mathscr{S}^{\prime}$ of order $\left(s^{\prime \prime}, t\right)$. By Thas [6], $s^{\prime} t \geq s^{2} t^{2}$, so by Haemers-Roos [2], $t^{3} \geq s \geq s^{\prime 2} t$, and hence $s^{\prime} \leq t$. Again by Thas [6] $t^{2} \geq s^{\prime} t \geq s^{\prime \prime 2} t^{2}$, so $s^{\prime \prime}=1, s^{\prime}=t$ and $s=t^{3}$.

Now let $p$ be as above and consider $\beta \in \mathscr{H}\left(p_{1}, p_{6}\right)$ mapping $p$ into any other point of $\mathscr{S}^{\prime}$ in $A^{*}\left(L_{1}\right)$. Again using Thas [6] one sees similarly as above that $\mathscr{S}^{\prime \beta}=\mathscr{S}^{\prime}$. Also every $\left(L, L^{\prime}\right)$-homology of $\mathscr{S}$ preserves $\mathscr{S}^{\prime}$ (granted $L$ and $L^{\prime}$ are opposite lines in $\mathscr{S}^{\prime}$ ). Hence $\mathscr{S}^{\prime}$ has transitive apartments and since $s^{\prime}=t, \mathscr{S}^{\prime}$ is Moufang by the first part of the proof. If $t=2$, then the result follows again by the uniqueness of the generalized hexagon of order $(8,2)$ (see Cohen-Tits [1]). So suppose $t>2$. Again by Thas [6], $\mathscr{S}$ does not contain a proper thick subhexagon with $s+1$ points on a line. So by Lemma $3, \mathscr{H}_{\left(p_{2}, p_{5}\right)}\left(p_{1}, L_{1}\right)$ is non-trivial and hence it contains a non-identity element $\sigma$. But restricted to any subhexagon of order $(t, t)$ containing $A, \sigma$ is a root-elation. Since every variety incident with $L_{1}, L_{2}$, $L_{4}, p_{1}$ or $p_{3}$ lies in such a subhexagon, it must be fixed by $\sigma$. Hence $\sigma$ is a root-elation of $\mathscr{S}$ and by Lemma 1 all long roots of $\mathscr{S}$ are transitive.

If some non-trivial element of $\mathscr{H}\left(L_{1}, L_{6}\right)$ fixes an element of $A^{*}\left(L_{2}\right)$, then it must fix a subhexagon of order $\left(s, t^{\prime}\right)$, implying $t^{\prime}=t$ by Thas [6] again. Hence $\mathscr{H}\left(L_{1}, L_{6}\right)$ (and also $\mathscr{H}\left(L_{3}, L_{4}\right)$ ) acts semi-regular on $A^{*}\left(L_{2}\right)$. By Lemma 4 , there exists a non-trivial $\sigma \in$ $\mathscr{H}_{L_{5}}\left(p_{1}, L_{1}\right)$ and by Lemma 5 we can choose $\sigma$ such that it also fixes every point incident with $L_{3}$. But now we can assume (by Lemma 1, e.g.) that $\mathscr{S}^{\prime}$ contains $p_{3}^{\sigma}$. Hence $\sigma$ is a root-elation in $\mathscr{S}^{\prime}$ and hence it fixes all lines through $p_{2}$ and $p_{4}$. So $\sigma$ is a root-elation and by Lemmas 1 and 2, all short roots of $\mathscr{S}$ are transitive. Hence $\mathscr{S}$ is Moufang.

Again it it clear by the proof that every root-elation is generated by generalized homologies. This completes the proof of the second case.

3.4. Case $s>t$ and $\mathscr{S}$ does not contain any subhexagon of or$\operatorname{der}\left(s^{\prime}, t\right), 1<s^{\prime}<s$. As in the previous case, $\mathscr{S}$ does not contain proper thick subhexagons with $s+1$ points on a line. Note that $t>2$. By assumption, $\mathscr{S}$ does not contain a proper thick subhexagon with 
$t+1$ lines through a point. So by (the dual of) Lemma $3, \mathscr{H}_{L_{5}}\left(p_{1}, L_{1}\right)$ is non-trivial. Also, similarly to the previous step, $\mathscr{H}\left(L_{3}, L_{4}\right)$ and $\mathscr{H}\left(p_{3}, p_{4}\right)$ both act non-trivially on $A^{*}\left(L_{2}\right)$. Hence by Lemma 5, $\mathscr{H}\left(p_{1}, L_{1}, L_{3}, p_{4}\right)$ is not trivial. Since $\mathscr{S}$ has no proper thick subhexagons with $s+1$ points on a line or $t+1$ lines through a point, the order of the group $\mathscr{H}\left(L_{1}, L_{6}\right)$, resp. $\mathscr{H}\left(p_{1}, p_{6}\right)$, is $t-1$, resp. $s-1$. Since $s>t$, there must be a $\left(p_{1}, p_{6}\right)$-homology acting non-trivially on $A^{*}\left(L_{2}\right)$. By Lemmas 5 and 1 , all short roots of $\mathscr{S}$ are transitive.

By Lemma $3, \mathscr{H}_{p_{5}}\left(p_{1}, L_{1}\right)$ is non-trivial and by Lemma 5 , $\mathscr{H}\left(L_{1}, p_{1}, L_{4}\right)$ is non-trivial. Let $\sigma \in \mathscr{H}\left(L_{1}, p_{1}, L_{4}\right)$. Then $\sigma$ acts semi-regularly on the set of $t$ lines through $p_{2}$ distinct from $L_{3}$. Hence $\sigma$ cannot act semi-regularly on the set $A^{*}\left(p_{3}\right)$ of size $t-1$. So $\sigma$ fixes at least three lines through $p_{3}$. By Lemmas 1 and 2, there exists $\tau \in \mathscr{H}\left(L_{1}, p_{3}, L_{4}\right)$ mapping $L_{3}$ into $L_{3}^{\sigma}$. But then $\sigma \tau^{-1}$ fixes $A$, it fixes all points incident with $L_{1}$ and it fixes at least three lines through $p_{3}$. By assumption, $\sigma \tau^{-1}$ is the identity and hence $\sigma=\tau$; hence $\mathscr{H}\left(L_{1}, p_{1}, p_{3}, L_{4}\right)$ is non-trivial.

Suppose $\mathscr{H}\left(L_{1}, p_{1}, L_{2}, p_{3}, L_{4}\right)=1$. This implies that the commutator

$$
\left[\mathscr{H}\left(p_{1}, L_{1}, p_{2}, L_{3}, p_{4}\right), \mathscr{H}\left(p_{3}, L_{2}, p_{1}, L_{1}, p_{2}\right)\right]
$$

(which is in general a subset of $\mathscr{H}\left(L_{1}, p_{1}, L_{2}, p_{3}, L_{4}\right)$ ) is also trivial and this implies geometrically that every element of $\mathscr{H}\left(p_{1}, L_{1}, p_{2}, L_{3}, p_{4}\right)$ fixes every line meeting $L_{1}$ or $L_{2}$.

Since $s>t$ there exists $\alpha \in \mathscr{H}\left(p_{1}, p_{6}\right)$ fixing at least three lines through $p_{2}$ and hence $\alpha$ fixes a subhexagon $S^{\prime}$ of order $(1, t)$. Clearly every element $\tau$ of $\mathscr{H}\left(L_{1}, p_{1}, p_{3}, L_{4}\right)$ stabilizes $\mathscr{S}^{\prime}$ and therefore $\tau$ fixes at least two points incident with every line through $p_{1}$ or $p_{3}$. Let $p \quad I \quad L \quad I \quad p_{1}$ and suppose $\tau$ fixes $p \neq p_{1}$. Then $\tau \in \mathscr{H}_{p}\left(p_{1}, p_{3}, L_{4}\right)$ and it is easy to see that $\mathscr{H}\left(L, p_{1}, p_{3}, L_{4}\right)=$ $\mathscr{H}_{p}\left(p_{1}, p_{3}, L_{4}\right)$. Hence $\tau$ fixes all points incident with $L$ (or use Van Maldeghem-Weiss [10] to conclude that in this case $\mathscr{S}$ is Moufang). So $\tau$ fixes every point collinear with $p_{1}$ or $p_{3}$. Now let $\sigma \in \mathscr{H}\left(p_{1}, L_{1}, p_{2}, L_{3}, p_{4}\right)$ be arbitrary and consider $\xi=\tau^{-1} \sigma \tau \sigma^{-1}$.

Suppose there exists a line $M I p_{2}$ such that $\sigma$ does not fix the unique point of $\mathscr{S}^{\prime}$ on $M$. Then we choose $\tau$ (of the preceding paragraph) such that $L_{3}^{\tau}=M$. Hence $p_{4}^{\xi} \neq p_{4}$. Let $P I$ $L \quad I \quad p_{1}$ with $p \neq p_{1}$ and $L \notin A^{*}\left(p_{1}\right)$. Consider the unique $\tau^{*} \in$ $\mathscr{H}\left(p, L, p_{1}, L_{1}, p_{2}\right)$ mapping $p_{4}^{\xi}$ to $p_{4}$. Then $\xi \tau^{*} \in \mathscr{H}_{p_{4}}\left(L, p_{1}, L_{1}, p_{2}\right)$. 
If $\xi \tau^{*} \neq 1$, then this would imply as above that $\mathscr{H}\left(L, p_{1}, L_{1}, p_{2}, L_{3}\right)$ is not trivial and hence by Lemma $1, \mathscr{S}$ would be Moufang. So we can assume that $\xi=\left(\tau^{*}\right)^{-1}$ and hence $\xi \in \mathscr{H}\left(p, L, p_{1}, L_{1}, p_{2}\right)$ and it fixes all points at distance two from $p_{1}$ except possibly those incident with $L_{2}$; it also fixes all lines through these points since $p$ and $L$ are arbitrary. So suppose $\xi$ does not fix all points incident with $L_{2}$. By Lemmas 1 and 2, all elements $\varphi$ of $\mathscr{H}\left(p_{1}, L_{1}, p_{2}, L_{3}, p_{4}\right)$ fix all points at distance two from $p_{2}$ except for some points on one unique line $L_{\varphi}$ through $p_{2}$. Since $t>2$, we can take a $\beta \in \mathscr{H}\left(L_{1}, L_{6}\right)$ not fixing $L_{\varphi}$. Then $\varphi^{\prime}=\beta^{-1} \varphi \beta \in \mathscr{H}\left(p_{1}, L_{1}, p_{2}, L_{3}, p_{4}\right)$ and $L_{\varphi^{\prime}} \neq L_{\varphi}$. But considering $\varphi \varphi^{\prime} \in \mathscr{H}\left(p_{1}, L_{1}, p_{2}, L_{3}, p_{4}\right)$, this leads to a contradiciton. Hence every $\varphi \in \mathscr{H}\left(p_{1}, L_{1}, p_{2}, L_{3}, p_{4}\right)$ fixes all points at distance two from $p_{2}$. Similarly one shows that every $\varphi \in \mathscr{H}\left(p_{1}, L_{1}, p_{2}, L_{3}, p_{4}\right)$ must fix every line at distance three from $p_{2}$. By Ronan [5], $\mathscr{S}$ is Moufang.

Suppose now $\sigma$ fixes on every line through $p_{2}$ the unique point of $\mathscr{S}^{\prime}$ distinct from $p_{2}$. Let $M$ be such a line and $p_{M}$ the corresponding point. Consider the unique $\sigma^{\prime} \in \mathscr{H}\left(p_{1}, L_{1}, p_{2}, M, p_{M}\right)$ mapping $p_{3}^{\sigma}$ back to $p_{3}$. Then $\sigma \sigma^{\prime} \in \mathscr{H}_{\left(p_{3}, p_{M}\right)}\left(p_{1}, L_{1}, p_{2}\right)$. So if $\sigma \sigma^{\prime} \neq 1$, then by Van Maldeghem-Weiss [10], $\mathscr{S}$ is Moufang. Hence we can assume that $\sigma=\sigma^{\prime}$. But that implies that $\sigma$ fixes all points of $M$ and all lines meeting $M$ since $\sigma^{\prime}$ does. Since $M$ was arbitrary, $\sigma$ fixes all lines at distance three from $p_{2}$, hence again by Ronan [5], $\mathscr{S}$, is Moufang.

Note however that this third case cannot occur since all Moufang generalized hexagons of order $(s, t)$ with $s>t$ have subhexagons of order $(t, t)$. This completes the proof of our main result.

4. Remarks. A similar theorem for generalized quadrangles follows immediately from Thas [7]. The finite thick generalized quadrangles with transitive apartments are the classical ones of order $(q, q)$, $\left(q, q^{2}\right)$ and $\left(q^{2}, q\right)$, for prime powers $q$. The classical generalized quadrangle $H\left(4, q^{2}\right)$ or order $\left(q^{2}, q^{3}\right)$ is only $(x, y)$-transitive for all pairs $(x, y)$ of opposite points. However, the result in Thas [7] is stronger than that. Indeed, for generalized quadrangles it is enough to require $(x, y)$-transitivity for all pairs $(x, y)$ of opposite points in order to conclude that the generalized quadrangle is Moufang.

As for the generalized octagons, they behave much like the $H\left(4, q^{2}\right)$ generalized quadrangle above with respect to generalized homologies. So there exists no finite thick generalized octagon with transitive apartments (and presumably neither an infinite one). 


\section{REFERENCES}

[1] A. M. Cohen and J. Tits, A characterization by orders of the generalized hexagons and a near octagon where lines have length three, European J. Combin., 6 (1985), 13-27.

[2] W. Haemers and C. Roos, An inequality for generalized hexagons, Geom. Dedicata, 10 (1981), 219-222.

[3] P. Fong and G. Seitz, Groups with a $(B, N)$-pair of rank 2, I and II, Invent. Math., 21 (1973), 1-57 and Invent. Math., 24 (1974), 191-239.

[4] W. M. Kantor, Generalized Polygons, SCABs and GABs, in Buildings and the Geometry of Diagrams (Ed. L. A. Rosati), Springer-Verlag, Berlin Heidelberg New York Tokyo, Como 1984, Lect. Notes in Math., 1181 (1986), 79-158.

[5] M. A. Ronan, A geometric characterization of Moufang hexagons, Invent. Math., 57 (1980), 227-262.

[6] J. A. Thas, A restriction on the parameters of a subhexagon, J. Combinatorial Theory Ser. A, 21 (1976), 115-117.

[7] _ The classification of all $(x, y)$-transitive generalized quadrangles, J. Combinatorial Theory, 42 (1986), 154-157.

[8] J. Tits, Sur la trialité et certains groupes qui s'en déduisent, Inst. Hautes Etudes Sci. Publ. Math., 2 (1959), 14-60.

[9] _ Classification of buildings of spherical type and Moufang polygons: a survey, in Teorie combinatorie (ed. B. Segre), Roma Accad. Naz. Lincei, (1976), 229-246.

[10] H. Van Maldeghem and R. Weiss, On finite Moufang polygons, preprint.

Received April 16, 1990 and in revised form November 19, 1990. The author is a Research Associate at the National Fund for Scientific Research (Belgium).

SEMINAR FOR GEOMETRY AND COMBINATORICS

STATE UNIVERSITY OF GENT

KRIJGSLAAN 281

9000 GeNT, BELGIUM 



\section{PACIFIC JOURNAL OF MATHEMATICS EDITORS}

\author{
V. S. VARADARAJAN \\ (Managing Editor) \\ University of California \\ Los Angeles, CA 90024-1555-05 \\ Herbert Clemens \\ University of Utah \\ Salt Lake City, UT 84112 \\ THOMAS ENRIGHT \\ University of California, San Diego \\ La Jolla, CA 92093
}

Nicholas ERcolani

University of Arizona

Tucson, AZ 85721

R. FINN

Stanford University

Stanford, CA 94305

VAUGHAN F. R. JONES

University of California

Berkeley, CA 94720

STEVEN KeRCKHOFF

Stanford University

Stanford, CA 94305

\section{C. MOORE \\ University of California \\ Berkeley, CA 94720}

MARTIN SChaRLEMANN

University of California

Santa Barbara, CA 93106

HAROLd STARK

University of California, San Diego

La Jolla, CA 92093

\section{ASSOCIATE EDITORS}

\begin{tabular}{|c|c|c|c|c|}
\hline ARENS & $\begin{array}{l}\text { E. F. BECKENBACH } \\
(1906-1982)\end{array}$ & NeumanN & $\begin{array}{l}\text { F. Wolf } \\
(1904-1989)\end{array}$ & K. Yoshida \\
\hline \multicolumn{5}{|c|}{ SUPPORTING INSTITUTIONS } \\
\hline \multirow{2}{*}{\multicolumn{2}{|c|}{$\begin{array}{l}\text { IVERSITY OF ARIZONA } \\
\text { IIVERSITY OF BRITISH COLUMBIA }\end{array}$}} & UNIVERS & Y OF OREGON & \\
\hline & & UNIVERS & OF SOUTHER & IIA \\
\hline \multicolumn{2}{|c|}{ LIFORNIA INSTITUTE OF TECHNOLOGY } & STANFO & UNIVER & \\
\hline \multirow{2}{*}{\multicolumn{2}{|c|}{$\begin{array}{l}\text { IVERSITY OF CALIFORNIA } \\
\text { ONTANA STATE UNIVERSITY }\end{array}$}} & UNIVERS & OF HAWAII & \\
\hline \multirow{2}{*}{\multicolumn{2}{|c|}{$\begin{array}{l}\text { ONTANA STATE UNIVERSITY } \\
\text { IIVERSITY OF NEVADA, RENO }\end{array}$}} & UNIVERS & YF TOKYO & \\
\hline & & UNIVERS & I OF UTAH & \\
\hline \multirow{2}{*}{\multicolumn{2}{|c|}{$\begin{array}{l}\text { W MEXICO STATE UNIVERSITY } \\
\text { EGON STATE UNIVERSITY }\end{array}$}} & WASHING & N STATE UNI & SITY \\
\hline & & UNIVERS & NASI & \\
\hline
\end{tabular}

The Supporting Institutions listed above contribute to the cost of publication of this Journal, but they are not owners or publishers and have no responsibility for its content or policies.

Mathematical papers intended for publication in the Pacific Journal of Mathematics should be in typed form or offset-reproduced (not dittoed), double spaced with large margins. Please do not use built up fractions in the text of the manuscript. However, you may use them in the displayed equations. Underline Greek letters in red, German in green, and script in blue. The first paragraph must be capable of being used separately as a synopsis of the entire paper. In particular it should contain no bibliographic references. Please propose a heading for the odd numbered pages of less than 35 characters. Manuscripts, in triplicate, may be sent to any one of the editors. Please classify according to the 1991 Mathematics Subject Classification scheme which can be found in the December index volumes of Mathematical Reviews. Supply name and address of author to whom proofs should be sent. All other communications should be addressed to the managing editor, or Elaine Barth, University of California, Los Angeles, California 90024-1555-05.

There are page-charges associated with articles appearing in the Pacific Journal of Mathematics. These charges are expected to be paid by the author's University, Government Agency or Company. If the author or authors do not have access to such Institutional support these charges are waived. Single authors will receive 50 free reprints; joint authors will receive a total of 100 free reprints. Additional copies may be obtained at cost in multiples of 50 .

The Pacific Journal of Mathematics (ISSN 0030-8730) is published monthly except for July and August. Regular subscription rate: $\$ 190.00$ a year (10 issues). Special rate: $\$ 95.00$ a year to individual members of supporting institutions.

Subscriptions, orders for numbers issued in the last three calendar years, and changes of address should be sent to Pacific Journal of Mathematics, P.O. Box 969, Carmel Valley, CA 93924, U.S.A. Old back numbers obtainable from Kraus Periodicals Co., Route 100, Millwood, NY 10546.

The Pacific Journal of Mathematics at P.O. Box 969, Carmel Valley, CA 93924 (ISSN 0030-8730) is published monthly except for July and August. Second-class postage paid at Carmel Valley, Californiä 93924, and additional mailing offices. Postmaster: send address changes to Pacific Journal of Mathematics, P.O. Box 969, Carmel Valley, CA 93924.

\section{PUBLISHED BY PACIFIC JOURNAL OF MATHEMATICS, A NON-PROFIT CORPORATION}




\section{Pacific Journal of Mathematics}

\section{Vol. 151, No. $2 \quad$ December, 1991}

Michael G. Eastwood and A. M. Pilato, On the density of twistor

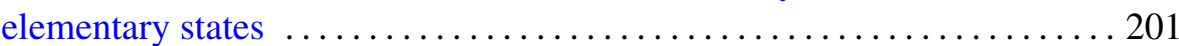

Brian E. Forrest, Arens regularity and discrete groups $\ldots \ldots \ldots \ldots \ldots \ldots 217$

Yu Li Fu, On Lipschitz stability for F.D.E ..................... 229

Douglas Austin Hensley, The largest digit in the continued fraction expansion of a rational number $\ldots \ldots \ldots \ldots \ldots \ldots \ldots \ldots \ldots \ldots \ldots . \ldots 237$

Uwe Kaiser, Link homotopy in $\mathbb{R}^{3}$ and $S^{3}$

Ronald Leslie Lipsman, The Penney-Fujiwara Plancherel formula for abelian symmetric spaces and completely solvable homogeneous

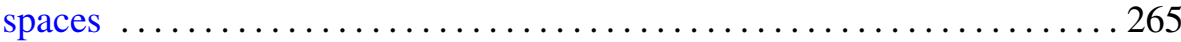

Florin G. Radulescu, Singularity of the radial subalgebra of $\mathscr{L}\left(F_{N}\right)$ and the

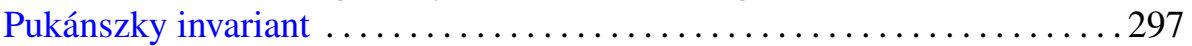

Albert Jeu-Liang Sheu, The structure of twisted SU(3) groups $\ldots . \ldots \ldots . \ldots 307$

Morwen Thistlethwaite, On the algebraic part of an alternating link . . . . . 317

Thomas (Toma) V. Tonev, Multi-tuple hulls .................. 335

Arno van den Essen, A note on Meisters and Olech's proof of the global asymptotic stability Jacobian conjecture ..................... 351

Hendrik J. van Maldeghem, A characterization of the finite Moufang hexagons by generalized homologies

Bun Wong, A note on homotopy complex surfaces with negative tangent bundles

Chung-Tao Yang, Any Blaschke manifold of the homotopy type of $\mathbf{C} P^{n}$ has the right volume 